\title{
LA CREACIÓN Y PROMOCIÓN DE EXPERIENCIAS EN UN DESTINO TURÍSTICO. UN ANÁLISIS DE LA INVESTIGACIÓN Y NECESIDADES DE ACTUACIÓN ${ }^{1}$
}

\author{
Rita Carballo Fuentes* \\ Sergio Moreno-Gil* \\ Carmelo León González* \\ J.R. Brent Ritchie***
}

\section{RESUMEN}

El artículo realiza una profunda revisión teórica de un término de creciente interés: la experiencia. Se aborda la comprensión de las dimensiones de la experiencia turística, cómo analizarlas, y cómo desarrollar y comunicar una experiencia turística, presentado especial atención a la marca y los eventos como paradigma promocional de la misma. Como aportaciones principales, este trabajo analiza e identifica la situación de la investigación sobre experiencias turísticas, y finaliza con una propuesta: matriz de diseño de la experiencia, para la implementación práctica de experiencias en un destino.

Palabras clave: experiencia turística, marketing de experiencias, investigación, marca, eventos, imagen.

Designing and promoting experiences in a tourist destination. An analysis of research and action needs

Recibido: 5 de noviembre de 2012

Devuelto para su revisión: 21 de mayo de 2013

Aceptado: 5 de septiembre de 2014

*Instituto de Turismo y Desarrollo Económico Sostenible -TiDES-. Cátedra UNESCO de Planificación Turística y Desarrollo Sostenible. Universidad de Las Palmas de Gran Canaria. E-mail: ritinaca@yahoo.es; smoreno@dede.ulpgc.es; cleon@daea.ulpgc.es.

**Chair, World Tourism Education and Research Centre. University of Calgary. 2500 University Drive NW. Calgary, Alberta, CANADA T2N 1N4.E-mail: britchie@ucalgary.ca

1 Los autores agradecen el apoyo financiero del Ministerio de Economía y Competitividad del Gobierno de España y la Subdirección General de Proyectos de Investigación. Proyecto ECO2012-35112. 


\begin{abstract}
This paper goes through a comprehensive literature review in order to properly deal with such an important topic as «the experience». This work examines the experience dimensions, how to analyze them, and how to develop and promote a tourist experience, paying attention to the brand and events as a paradigmatic promotional tool. The main contribution of this paper focus in identifying state of the art about tourist experiences research, and it shows an operative framework to put into practice the tourist experience in a destination.
\end{abstract}

Key words: tourist experience, experiential marketing, research, brand, events, image.

\title{
1. INTRODUCCIÓN
}

La experiencia en turismo es un tema que ha sido ampliamente usado, tanto en el entorno académico como profesional. Sin embargo, su conceptualización, explicación, metodologías de análisis, y esquemas de pensamiento y aplicación para su diseño y promoción, son aspectos que no han recibido gran atención. Este trabajo pretende aportar algo de conocimiento académico y líneas de aplicación práctica para el correcto desarrollo de experiencias, tan buscadas y necesitadas en los destinos turísticos.

El turista que viaja a un destino turístico no realiza meramente un viaje físico. El verdadero viaje es interior, y radica en cómo percibe el lugar que visita, sus gentes, y el impacto que esta percepción subjetiva y personal le produce. En función de múltiples factores y condicionantes, el turista vive una experiencia vacacional. El viaje a un destino turístico es siempre una experiencia para el turista, de mayor o menor significado, pero una experiencia. Las experiencias se producen cuando los consumidores buscan un producto, cuando lo compran y reciben el servicio, y cuando lo consumen (Brakus, Schmitt, y Zhang 2008; y Holbrook, 2000). Además, los turistas están deseosos de pagar un mayor precio por una experiencia cautivadora (Mehmetoglu y Engen, 2011). Por consiguiente, desde un enfoque más conceptual debemos plantearnos ¿qué es una experiencia turística?, y ¿cómo comprenderla y qué técnicas usar para su análisis e investigación? Posteriormente, y con profundas implicaciones prácticas, también debemos abordar cómo desarrollar adecuadamente una experiencia y cómo comunicarla, incluyendo el impacto de las actividades promocionales como, por ejemplo, es el caso de los eventos.

Este trabajo pretende proveer de un marco que facilite la identificación y mejora de la comprensión de los principales desafíos a los que nos enfrentamos en la investigación de la experiencia del turista y su puesta en práctica. Estos desafíos tienen una dimensión tanto teórica como de gestión. El artículo comienza con un resumen del examen de la literatura actual en este campo, fundamentado en el trabajo seminal de los profesores Ritchie y Hudson (2009), que identifica el conocimiento existente de grandes corrientes de pensamiento teórico y de investigación empírica. Posteriormente, se centra en la comunicación de la experiencia a través del denominado marketing de experiencias, y desarrolla el proceso de comunicación de la misma a través de la marca (aglutinador central de la comunicación) y los eventos (paradigma de la creación y comunicación de experiencias). Finalmente, a 
modo de cierre, el artículo aborda una sintética explicación práctica y metodológica para el diseño de experiencias turísticas de éxito en un destino turístico: la matriz de diseño de la experiencia.

\section{EL CONCEPTO DE EXPERIENCIA TURÍSTICA Y SU METODOLOGÍA DE ANÁLISIS}

Aún no se ha llegado a una definición unánime de experiencia, pero es sobre todo un acontecimiento personal, a menudo cargado de un significado emocional importante, basado en la interacción con los estímulos que son los productos o servicios consumidos (Holbrook y Hirschman, 1982). Este acontecimiento puede incluso llevar al individuo a definir las experiencias como extraordinarias (Arnould y Price, 1993). En la investigación de Harrison (2001) sobre cómo los turistas recrean sus experiencias significativas, se señala que son aquellas donde «los turistas se involucran más de lo que normalmente se espera» (nunca seré el mismo... he cambiado). Como uno de sus informantes más elocuentes describió:

[El viaje ofrece] la oportunidad, aunque sea de forma accidental, de encontrarse con partes de uno mismo que ni siquiera sabía que existía. En cierto sentido es como si una parte de ti se hubiese quedado dormida y de repente se vuelve a despertar. Viajando a otros lugares tengo la oportunidad de descubrir parte de mí por la forma en que actúo ante algo extraño (2001:167).

El detonante del gran interés en la literatura sobre la experiencia turística, y más concretamente, las experiencias auténticas, puede remontarse a los primeros trabajos de autores como Boorstin, MacCannell, y Cohen. Los turistas no se satisfacen fácilmente con un evento artificial, sino que más bien buscan la autenticidad de las experiencias. Cohen (1979) define la experiencia turística como la relación entre la persona y su visión del mundo dependiendo de la ubicación de su centro de referencia con respecto a la sociedad a la que pertenece. Ryan (1995), Aho (2001), Berry, Carbone y Haeckel (2002), Jennings y Nickerson (2006) y IACVB (2005) han señalado que la satisfacción y la calidad por si solos, ya no describen adecuadamente la experiencia que busca el turista en la actualidad. En el intento de definir y entender la esencia de la experiencia turística existe una secuencia de sub-corrientes en la que los investigadores utilizan como punto de partida diferentes marcos teóricos específicos. Csikszentmihalyi (1975) fue un pionero en este tema con su trabajo «la psicología de la experiencia óptima», donde describe la importancia de experiencias provistas de un sentido de alegría, una profunda sensación de diversión que se desea perdure y que se convierte en la memoria en un hito de lo que debería ser la vida. Esto lo denomina, la «experiencia óptima». Csikszentmihalyi (2000) aplicó sus conocimientos psicológicos al comportamiento del consumidor y el marketing, destacando que el consumo es el comportamiento por el cual la entropía se incrementa a cambio de experiencias o recompensas experienciales. Somos seres sociales, y queremos contar lo que hemos aprendido de nuestras experiencias. 
Abrahams (1986) asegura que la experiencia señala nuestro deseo de tener actos auténticos, a expensas de los autorizados, ya que apreciamos más aquellos momentos que podemos decir que «fueron grandes», pero que se nos acercan y nos cogen por sorpresa, en los que las expectativas se ven superadas como ocurre en el caso de las primeras experiencias, que en caso de éxito, son tan sorprendentes porque hemos oído hablar de ellas, e incluso hemos hablado sobre ellas, pero consiguen sorprendernos de todos modos. Holbrook y Hirschman (1982) claman por pasar el marketing del mundo del producto al mundo de la experiencia, entendiendo el comportamiento del turista, no sólo como de procesamiento de información, sino esencialmente experiencial, sin ignorar el carácter placentero de las actividades de ocio y la importancia de los disfrutes sensoriales, los sueños, el goce estético, y las respuestas emocionales.

Por otra parte, Havlena y Holbrook (1986) aplicaron las dimensiones del modelo de Mehrabian y Russell (1974) y encontraron tres factores: el placer, la excitación y el dominio, que pueden ser utilizados para describir con éxito el carácter emocional de las experiencias de consumo. El placer se refiere al grado en que una persona se siente bien, feliz, o satisfecho por la situación; la excitación se refiere al grado en que una persona se siente estimulada; y el dominio se refiere a la medida en que el individuo se siente con el control de la situación.

De forma específica, Arnould y Price (1993) en su investigación sobre la actividad del rafting, identificaron tres dimensiones claves de una experiencia extraordinaria: i) el contacto con la naturaleza; ii) la creación de comunidad con amigos, familiares e incluso desconocidos; y iii) el crecimiento personal y la renovación de uno mismo. Estos tres temas juntos, son significativos para la explicación de la satisfacción global.

Otto y Ritchie (1996) identificaron seis dimensiones fundamentales de la construcción de la experiencia: una dimensión hedonista, una dimensión social o interactiva, una búsqueda de la novedad o dimensión de escape, una dimensión de confort, una dimensión de seguridad, y una dimensión de búsqueda de estímulos o retos. Así, los que buscan ofrecer a los turistas una experiencia de calidad deberían considerar la forma de proporcionar a los visitantes cada uno de estos seis componentes de la experiencia turística.

Pine y Gilmore (1998) destacaron cuatro dimensiones de la experiencia, las cuales clasificaron como: estética, educacional, de entretenimiento y escapismo. Aho (2001) distingue entre cuatro núcleos esenciales de la experiencia turística: experiencias emocionales, aprendizaje, experiencias prácticas, y experiencias transformadoras.

La motivación es otro de los componentes de la experiencia, es una mezcla compleja de escapismo, socialización y auto-realización (Ryan, 1997). Esto ayuda a explicar el creciente interés en los deportes participativos y extremos (Arnould y Price, 1993), y en los nuevos tipos de turismo de aventura, cultural, deportivo y creativo (Richards y Wilson, 2006).

Por otra parte, O’Dell y Billing (2005) analizaron el concepto de experiencia-paisaje, donde las experiencias que se gestionan y se consumen se pueden comparar con paisajes estilizados que son diseñados y planificados estratégicamente. Son espacios en los que diversos grupos se mueven y se ponen en contacto unos con otros: paisajeindustrial, paisaje-regional, paisaje-arquitectura, y paisaje-nostalgia. Así, estos paisajes sólo recrean una atmósfera para la experiencia, dado que hay límites a la planificación 
y su diseño, si tenemos en cuenta tres características de las experiencias en turismo: (1) Las experiencias surgen de los orígenes sociales y culturales de las personas. Dado que los diferentes orígenes dan lugar a interpretaciones diversas para un solo producto turístico, ¿cómo puede un único producto interesar y emocionar a todos los clientes? (2) Las experiencias tienen múltiples facetas. Surgen de las actividades y el entorno físico, así como del significado social integrado en las actividades. Las personas tienen experiencias diferentes, incluso si están haciendo lo mismo en el mismo lugar; y (3) Las experiencias son existenciales y están encarnadas en las personas, ya que son personales y únicas. En resumen, los factores sociológicos, psicológicos y contextuales, determinan el resultado de la experiencia, existiendo así condicionantes de la experiencia turística que pueden ser clasificados como: tiempo, dinero, conocimientos, habilidades y actitudes que dependen de los individuos.

Sin embargo, el análisis actualmente predominante en el turismo aún se basa en el paradigma de procesos de información cognitivos. Se busca primero entender el papel funcional de la toma de decisiones basado en la adquisición de información sobre el viaje y, posteriormente, la exploración de información suplementaria mediante la cual el turista recrea una imagen o estética. Se hace patente la necesidad de continuar investigando las experiencias de los turistas en sus diversas dimensiones, enfrentándonos aquí con el reto de la metodología a aplicar en el estudio y análisis de las experiencias turísticas.

Una vez contextualizado el término y analizadas sus dimensiones, podemos concluir que la experiencia turística se trata de un proceso acumulativo, por etapas, que incluye la fase anterior, vacacional en el destino, y posterior a las vacaciones, y que se necesita de una preparación explícita para que suceda. Al tratarse de una percepción subjetiva, es absolutamente necesario incorporar al turista en dicho proceso, y se han identificado algunas dimensiones específicas que debieran integrase en el análisis, si bien la evolución del análisis académico continuará identificando otras posibilidades. Las principales dimensiones identificadas son: involucración del turista, integrando los elementos del entorno externos al turista con los internos del individuo, siendo la naturaleza un facilitador clave; el shock sensorial, apelando a los cinco sentidos y generando una excitación y estimulando al turista, creando sorpresa y un entorno de escape; autenticidad, vinculando lo vivido con el entorno inmediato y el destino (sense of place); diversión, generando placer y un entorno agradable y de entretenimiento; sociabilidad, proporcionando la posibilidad de compartir y de participar en una comunidad; personalización, que aporta libertad y control, donde el turista siente que puede elegir y definir en cierta medida gracias a su habilidad y esfuerzo el resultado final, consiguiéndolo por el mismo; y autodescubrimiento y trasformación, donde a través de un aprendizaje y un intercambio de valores y reflexiones, el turista siente un cierto cambio.

\subsection{Metodologías específicas utilizadas en la investigación de experiencias en turismo}

Debido a que la mayoría de experiencias en turismo tienen lugar en un corto periodo con episodios ininterrumpidos, en vez de ocurrir en largos períodos de tiempo, puede ser difícil alcanzar una verdadera comprensión de las mismas. La mayoría de las investigaciones se han centrado en métodos mixtos para estudiar la experiencia de los participantes, 
como el trabajo de Arnould y Price (1993), que analizan la experiencia antes, durante y después del viaje. Por otra parte, Nickerson et al. (2004) trataron de comparar los significados después de las experiencias de los visitantes con tres enfoques metodológicos: diario de composición abierta, envío de encuestas por correo y las entrevistas en profundidad. Los tres enfoques proporcionan información sobre las dimensiones de la experiencia y la comprensión sobre la relación con el medio y las actividades, pero sólo las entrevistas en profundidad permitieron obtener una conexión espiritual con la experiencia vacacional.

Otro ejemplo de métodos mixtos es el estudio de Lee et al. (1994) cuyos datos se recogieron en dos etapas. La primera mediante la colección inmediata de experiencias de ocio recordadas a través de SITRM (método de cinta de grabación por iniciativa propia), que requiere que los participantes usen localizadores electrónicos y lleven diarios de auto cumplimentación que generalmente emplean encuestas cuantitativas. La segunda etapa se diseñó para evaluar la experiencia a través de entrevistas en profundidad. El análisis de los datos reveló una serie de características de la experiencia de ocio, algunas de las cuales fueron ya previamente identificadas en la literatura: la vinculación social, la comunión con la naturaleza, etc.

Un ejemplo de análisis de características positivas y negativas en la experiencia se encuentra en el trabajo de Jackson et al. (1996), quienes utilizaron la Técnica de Incidentes Críticos en la que se les pide a los turistas enumerar y describir sus experiencias más positivas y negativas. Este marco conceptual permitió a los investigadores determinar cuáles de las cuatro causas los turistas suelen usar para la explicación de sus experiencias: habilidad, esfuerzo, facilidad de la tarea, y la suerte. Los resultados del estudio indicaron que los turistas eran más propensos a atribuir la causa de las experiencias positivas a ellos mismos, y más propensos a atribuir la causa de sus experiencias negativas a factores externos a modo de auto-protección. Estas atribuciones externas incluyen a las empresas proveedoras, la población local y simplemente la mala suerte.

Con el fin de profundizar en las respuestas de interpretación de las experiencias de los turistas, se pueden utilizar otra serie de técnicas, como las fotografías de paisajes, que permiten analizar cómo los diferentes grupos de turistas eligen diferentes experiencias en la misma ubicación geográfica. También la técnica de elicitación de metáforas de Zaltman (ZMET) que permite extraer de los turistas modelos mentales de su experiencia vacacional donde los participantes proporcionan imágenes y metáforas que representan sus pensamientos y sentimientos acerca de las vacaciones.

Un aspecto importante a considerar en la metodología de comprensión y medición de las experiencias es cómo tratar de minimizar el sesgo por olvido de los recuerdos, y el sesgo en el estado de ánimo (Larson y Csikszentmihalyi, 1983). Así, es necesario analizar la relación existente entre la memoria y las experiencias. Este vínculo no es nuevo, y como Cutler y Carmichael (2010) señalaron se remonta a los primeros trabajos de la psicología medioambiental (Fridgen, 1984). Desde entonces, distintos autores han investigado la influencia de la experiencia turística con relación a los componentes cognitivos (Pearce y Foster, 2007), afectivos (Noy, 2004) y cambios psicomotrices a nivel individual (Arnould y Price, 1993).

Otros autores han empleado la Memoria de Trabajo, donde los recuerdos son los datos en bruto, y el sujeto y el objeto de la investigación se conviertan en uno, el investigador 
y el investigado son co-investigadores, hay interpretación colectiva y teorización de los recuerdos, y el enfoque colectivo permite la posibilidad de la liberación. Se diferencia entre la fenomenología (el estudio de lo que las personas perciben del mundo) y fenomenografía (el estudio de la forma en que perciben el mundo). Es evidente que el análisis de las experiencias requiere de nuevas formas de investigación como las del análisis de los momentos críticos de la verdad (Carlzon, 1987), la creación de un mapa de experiencia (Schmitt, 2003) o el desarrollo de un guión teatral (Harris et al, 2003). Así, la investigación etnográfica y narrativa (e.g., Arnould y Price, 1993) se presenta como una línea de trabajo que necesita un mayor desarrollo sobre la experiencia turística.

Finalmente, algunos autores han optado por utilizar una escala cuantitativa para trazar las dimensiones de la experiencia. Obviamente, este análisis presenta las ventajas de cualquier método cuantitativo, pero parece limitado en alcance si se desea comprender en profundidad la naturaleza de las experiencias turísticas y su valoración. Por consiguiente, parece que el acompañamiento con otras metodologías como las expuestas anteriormente, favorece un acercamiento más acertado a la naturaleza de las experiencias turísticas. La conocida naturaleza intangible de las mismas, con simultaneidad producción-consumo, duración limitada y heterogeneidad, refuerzan este planteamiento de integrar diversas metodologías de análisis.

En la empresa y destinos turísticos, el proceso de desarrollo de experiencias memorables para y con los clientes es un medio esencial para la creación de valor superior y una ventaja competitiva (Voss, 2004). Como conclusión, parece adecuado combinar el uso de metodologías mixtas, siempre incluyendo técnicas cualitativas, y los resultados de los análisis previos realizados con estas técnicas parecen confirmar las dimensiones resumidas en el apartado anterior. En cualquier caso, y con independencia de la metodología de análisis, queda patente la necesidad de recrear una experiencia memorable para el turista y comunicarla adecuadamente. En este sentido, la marca es la que garantiza esa promesa de experiencia memorable. A continuación se profundizará en este aspecto crucial a través del análisis de la marca de destino.

\section{LA PROMOCIÓN DE UN DESTINO Y UNA EXPERIENCIA MEMORABLE}

En un contexto turístico, el destino puede ser considerado como el escenario para la experiencia del turista. Para entender la experiencia del turista y la comunicación de la marca de destino de experiencias, el destino debe de ser definido desde tres perspectivas complementarias: económica, física y representativa. El cuadro 1 muestra las diferencias de enfoque de estas perspectivas. Desde una perspectiva más económica, autores como Camisón y Monfort (1998) y Keiser (1998) han definido el destino como una agrupación de diferentes organizaciones multiactivas, asociadas sobre un soporte espacial claramente diferenciado, que comercializa un producto turístico integrado. Desde un enfoque más físico, el destino es el espacio geográfico-temporal en el que se desarrolla la experiencia turística (Goodall y Asworth, 1988). Desde el tercer enfoque de destino, la perspectiva representativa, el destino es definido como la razón de ser del viaje, y una combinación de elementos tangibles e intangibles diseñados para satisfacer las necesidades de los turistas (Cooper et al., 1989), que estos consumen bajo el nombre de marca de un des- 
tino. Siguiendo a Bigné et al. (2000), el destino es un concepto percibido, variando la concepción de destino de una persona a otra. Por consiguiente, la marca se convierte en un elemento central de la experiencia del turista en el destino.

Cuadro 1

DIFERENCIAS ENTRE LAS PERSPECTIVAS DE DEFINICIÓN DE DESTINO TURÍSTICO

\begin{tabular}{lccc}
\hline & $\begin{array}{c}\text { PERSPECTIVA } \\
\text { ECONÓMICA }\end{array}$ & $\begin{array}{c}\text { PERSPECTIVA } \\
\text { FÍSICA }\end{array}$ & $\begin{array}{c}\text { PERSPECTIVA } \\
\text { REPRESENTATIVA }\end{array}$ \\
\hline Énfasis & $\begin{array}{c}\text { Las relaciones } \\
\text { económicas que tienen } \\
\text { lugar }\end{array}$ & $\begin{array}{c}\text { El lugar o territorio en el } \\
\text { que acontece el turismo }\end{array}$ & $\begin{array}{c}\text { La experiencia del turista } \\
\text { y su representación } \\
\text { mental }\end{array}$ \\
\hline $\begin{array}{l}\text { Objetividad } \\
\text { del concepto }\end{array}$ & Objetiva & Objetiva & Subjetiva \\
\hline $\begin{array}{l}\text { Consideración } \\
\text { de la imagen }\end{array}$ & $\begin{array}{c}\text { Elemento secundario, } \\
\text { vinculado a la promoción }\end{array}$ & $\begin{array}{c}\text { Es un atributo que da } \\
\text { nombre e identifica el } \\
\text { espacio físico }\end{array}$ & $\begin{array}{c}\text { Es un componente } \\
\text { básico y ligado al propio } \\
\text { destino }\end{array}$ \\
\hline
\end{tabular}

Fuente: Elaboración propia.

Continuando con la perspectiva representativa, los turistas tienen imágenes estereotipadas de los diferentes destinos. Muchos destinos en todo el mundo se venden de manera muy similar, y su imagen gira en torno a los iconos más usuales, como naturaleza, playas, diversión o adecuación para familias o parejas. Los mensajes suelen ser genéricos, normalmente centrados en la idea del escapismo y descubrimiento. Sin embargo, algunos destinos sí han conseguido desarrollar un único y claro posicionamiento de marca, basado en la experiencia del turista en el destino más que en los atributos físicos del mismo, captando la atención del turista hacia un destino que es percibido como deseado e irresistible (Blain et al., 2005).

Hoy en día, la mayoría de los destinos afirman tener paisajes espectaculares, excelentes lugares de interés, gente amable, y una cultura y patrimonio histórico único. Sin embargo, estos factores ya no son diferenciadores, y un destino fundamenta su éxito en el potencial de reducir la posibilidad de ser sustituido por otros. Para lograr esto, el destino se debe preocupar más por la experiencia del turista, creando un marketing que afecte a las emociones de los clientes potenciales (Ritchie y Hudson, 2009).

La imagen de un destino influye en los procesos de selección de los turistas, en la evaluación de dicho destino y en las intenciones futuro (Bigné et al., 2001). Los turistas constituyen sus expectativas en base a la información que transmite la imagen del destino. Así, un destino turístico sólido y competitivo necesita apoyarse en un símbolo que lo identifica y lo comunica y posiciona en base a sus atributos (Obiol, 2002). La definición más común y aceptada de la imagen de marca es «las percepciones a las que se ve asociada una marca en la mente del consumidor» (Keller, 1993). Así, la imagen de marca es multidimensional (Martínez y Chernatony, 2004) ya que se compone de los beneficios funcionales y simbólicos de la misma (Low y Lamb, 2000). Kotler (1997) define la marca 
como «un nombre, término, signo, símbolo, diseño o combinación de ellas que tiene por objetivo identificar los bienes y servicios de un vendedor o grupo de vendedores para diferenciarlos de los de la competencia»

La literatura general del marketing y la marca (e.g., Aaker, 1991) y la literatura de marca de destino (e.g., Ritchie y Ritchie, 1998; Hankinson, 2004) definen la marca de destino como el conjunto de actividades de marketing que (1) apoya la creación de un nombre, símbolo, logotipo, palabra o gráfico que fácilmente identifica y diferencia un destino, que (2) constantemente transmite la promesa de una experiencia memorable que esta únicamente asociada a ese destino, que (3) sirve para consolidar y reforzar la conexión emocional entre el visitante y el destino, y que (4) reduce el coste de búsqueda del consumidor y el riesgo percibido. La eficacia de la marca de un destino requiere de una estrategia de venta única que sea sostenible, creíble y relevante, y que la competencia quiera y tal vez sea capaz de imitar, pero que no pueda superar o usurpar (Morgan, Pritchard y Piggott, 2002:21).

El reconocimiento de la marca reduce la necesidad de búsqueda de información detallada y contribuye a que un destino se convierta finalmente en el elegido por el visitante (Seddighi y Theocharous, 2002). Por consiguiente, comprender el contenido y la estructura del reconocimiento de una marca es importante porque ambos influyen en lo que le viene a la mente al consumidor cuando piensa en dicha marca.

Se puede concluir que el análisis de la experiencia conduce a una concepción del destino desde una perspectiva representativa donde la marca es un elemento clave en la identificación de dicha experiencia y, por consiguiente, la confección de la marca debe estructurase desde el análisis de la experiencia del turista. A continuación se presenta un marco conceptual para entender el proceso de construcción de la marca. Posteriormente se describe el cambio en el enfoque de marca que necesitan los destinos, lo que implica centrarse en la experiencia turística, creando un marketing basado en estas experiencias para influir en las emociones de los viajeros potenciales.

\subsection{Creación de la marca de un destino turístico de experiencias}

Muchas contribuciones relevantes se han desarrollado recientemente en la literatura sobre la marca, incluyendo la personalidad de la marca, la comunidad de la marca, la confianza de la marca, el apego a la marca, y el amor a la marca (Aaker, 1997; Carroll y Ahuvia, 2006; Delgado-Ballester, Munuera-Alemán y Yagüe-Guillén, 2003; McAlexander, Schouten y Koenig, 2002; Thomson, MAcInnis y Park, 2005). Desarrollar la marca de un destino de experiencias puede incluir múltiples atributos subyacentes en dicho concepto. Así, diferentes autores destacan por ejemplo la personalidad de la marca (Ekinci y Hosany, 2006), la imagen (Cai, 2002), y los atributos (Blain et al., 2005), pero todavía existe una gran confusión entre los académicos y la industria sobre las definiciones de cada elemento, dándose una falta de consenso en cuanto a la forma en que estos colaboran para formar una verdadera marca de destino (Tasci y Kozak, 2006). Siguiendo a Aaker (1991), podemos definir el valor de la marca del destino como los activos (o pasivos) de la marca vinculados al nombre de un destino que siempre añaden (o restan) servicios o experiencias. Además, el valor de la marca de un destino turístico es creado por una combinación de elementos: 
el reconocimiento del nombre del destino, la calidad percibida del destino, las asociaciones a la marca del destino, la fidelidad al destino, y otros activos como la ventaja competitiva creada por la marca.

Hasta la fecha, la mayor parte de las investigaciones sobre experiencias se ha centrado en los atributos del producto y la categoría de las experiencias, y no en las experiencias proporcionadas por la marca. La experiencia de marca se define como sensaciones, sentimientos, conocimientos y respuestas de comportamiento provocadas por estímulos relacionados con la marca que forman parte del diseño e identidad de la misma, el embalaje, la comunicación y el entorno (Brakus et al., 2009).

Según Alloza (2008), la experiencia de la marca se puede definir como la percepción de los consumidores que en todo momento tienen contacto con la marca, ya sea cuando reciben imágenes publicitarias, durante el primer contacto personal, o durante el trato personal recibido. La experiencia de marca se crea cuando los clientes utilizan la marca, hablan a otros de la marca, buscan información sobre la marca, promociones, eventos, y otros (Ambler et al, 2002).

En contraste con la satisfacción del cliente, la experiencia de la marca no sólo se produce después del consumo, sino que se produce independientemente de que exista una interacción directa o indirecta con la marca (Hudson, Wang y Moreno-Gil, 2011). Por otra parte, una marca de experiencia no tiene que ser sorprendente, ya que puede ser ambas cosas; esperada o inesperada (Brakus et al, 2009).

El proceso de desarrollo de la marca del destino experiencial (Knapp y Sherwin, 2005; Morgan et al, 2003) se puede estructurar desde el punto de vista estratégico en cuatro sencillos pasos: (1) evaluar la situación actual del destino y sus valores, (2) desarrollar una identidad y promesa de marca, (3) comunicar esa promesa, y finalmente (4) medir la eficacia de la marca.

La primera etapa en la creación de una marca de destino experiencial es realizar un diagnóstico y establecer los valores fundamentales del destino y su marca. Esta etapa debe considerar la importancia de la marca para el turista actual y cómo se compara con los competidores directos. Es necesario incorporar un punto de vista objetivo como es el caso de las perspectivas de los visitantes actuales y potenciales, incluyendo las diferencias entre los distintos segmentos; así como a otros agentes como los prescriptores, los organizadores de eventos, y los tour operadores, con el fin de obtener un análisis independiente de la situación actual del mercado (Knapp y Sherwin, 2005).

Una vez que la investigación de mercado se ha completado, el siguiente paso es el desarrollo de la identidad de marca. Es fundamental para el éxito de cualquier marca de destino encontrar la medida en que la personalidad de la marca del destino interactúa con el mercado objetivo. La personalidad de la marca tiene dos componentes: la cabeza y el corazón. La «cabeza» es la característica lógica, mientras que el «corazón» es el beneficio emocional y sus asociaciones (Morgan et al., 2003). La propuesta de marca y su comunicación puede estar basada en torno a ambos componentes, aunque hay un creciente interés en el último. Estos atributos emocionales y funcionales subyacen en el concepto de promesa de marca, y cada destino debe comunicar a los visitantes actuales y potenciales los beneficios y experiencias que pueden esperar a su llegada (Knapp y Sherwin, 2005). 
Siguiendo a Ritchie y Hudson (2009), los destinos turísticos se han dado cuenta de que la promesa de marca tiene que ir más allá de los aspectos físicos de un destino, y crear una expectativa de experiencia a la llegada del visitante. Estos autores utilizan varios ejemplos como la promesa de Las Vegas, que es una experiencia excitante, sexy y peligrosamente segura «safely dangerous». La promesa de visita de Irlanda es la entrega e involucración de la gente, la riqueza, el colorido, el entorno virgen, y el medioambiente natural y cultural. Los lugareños también se encuentran en el objetivo de la promesa de marca de Australia, sugiriendo una bienvenida más cálida, distintiva y auténticamente australiana. A los visitantes de Nueva Zelanda se les promete descubrimiento personal, y experiencias más auténticas y genuinas que las que ya conocen, todo ello con el telón de fondo de los impresionantes paisajes. Finalmente, la India promete una oportunidad única para el fortalecimiento físico, el rejuvenecimiento mental, el enriquecimiento cultural y la elevación espiritual.

El tercer paso para la comunicación de la promesa de marca, exige que la esencia de la marca se comunique de forma integral, y por parte de todos los agentes, incluyendo también la realización de diversas campañas promocionales, el logo de la marca, lema, la historia y el nombre (Ritchie y Hudson, 2009). Así, por ejemplo, para la campaña «Lo que pasa en Las Vegas se queda en Las Vegas» la ciudad desarrolló una serie de innovadores anuncios de televisión que no radicaban en imágenes típicas de neón, showgirls y juegos de azar, sino más bien en recrear un concepto actualizado de Las Vegas como el lugar para realizar los sueños, ambiciones secretas y fantasías, y al que el turista no regresará. La sexualmente sugerente y divertida serie de anuncios, atraía por igual a hombres y mujeres de diferentes edades, que votaron por los anuncios más atractivos durante un año en el periódico «USA Today». La dirección del sitio web, al final de cada anuncio, proporcionaba también más información y un sistema de fácil reserva.

La etapa final de la creación de una marca de destino es evaluar el rendimiento que la misma ha tenido en el mercado: la medición de su eficacia. La continua evaluación de la comunicación es la clave, al igual que también lo es tener una mente abierta y la voluntad de aceptar el cambio por parte de los gestores de la marca (Ritchie y Ritchie, 1998). El secreto es evolucionar continuamente y enriquecer la marca original, fortalecer la base inicial aumentando su atractivo y ampliando el mercado.

En cualquier caso, solo medir la llegada de turistas es una forma de medir la eficacia, aunque muy deficiente y en la literatura ya existen numerosos métodos de valoración de la imagen de un destino (Serarols y Royo, 2005). La forma más adecuada de analizar el rendimiento de una marca de destino es controlar la eficacia de la marca en lugar de la eficacia de las campañas individuales (Wells et al., 2006), realizando una valoración económica de la marca y los atributos de la imagen, a través de las diferentes técnicas existentes (Carballo et al., 2011) siendo capaz de identificar los elementos de la experiencia que realmente aportan valor.

Se puede concluir que la marca, no sólo incorpora las experiencias del turista en el destino para su creación, sino que se trata de un proceso dinámico, donde la propia marca también aporta una experiencia al turista, existiendo una metodología estructurada que permite el proceso de desarrollo de la marca y su contribución a un marketing de experiencias. 


\subsection{El marketing de experiencias y la contribución de los eventos al marketing de experiencias}

Generar una marca de destino experiencial no es suficiente, ya que debe comunicarse adecuadamente, siendo necesario integrar un marketing de experiencias. Siguiendo a Presenza et. al (2007) y Ritchie y Hudson (2009), se trata de un enfoque relativamente nuevo y que contrasta con el marketing tradicional. Mientras el marketing tradicional se centra en considerar que los clientes toman decisiones racionales basadas en las características funcionales y los beneficios que ofrece el producto; el marketing de experiencias se focaliza en considerar a los clientes como seres emocionales, centrados en la consecución de experiencias placenteras (Williams, 2006). De acuerdo con autores como Schmitt (1999), el marketing de experiencias describe el punto de compromiso entre una marca y su consumidor. Si se ejecuta correctamente, genera a corto plazo cambios de comportamiento y establece una conexión emotiva que crea una relación profunda y, finalmente, una respuesta racional a la compra de la marca y el producto (Robertson, 2007). Se argumenta que tal y como evoluciona la ciencia del marketing, el marketing de experiencias se convertirá en la herramienta dominante del futuro (Williams, 2006). Schmitt (1999) fue de los primeros que sugerían que las experiencias pueden atraer los sentidos y emociones de los consumidores de una manera memorable. El marketing de experiencias reconoce que el interés de los consumidores no se limita a los beneficios puramente funcionales, sino al consumo de una experiencia total (Leighton, 2007). Esta experiencia tendrá un efecto positivo en las emociones y, posteriormente, en la intención de comportamiento (Tsaur et al., 2006).

Pero el marketing de experiencias no sólo significa tener una oferta de experiencias. La experiencia debe ser comercializada efectivamente como tal (Petkus, 2004). Además de comunicar lo obvio, las campañas promocionales necesitan acercar la marca hacia los consumidores deslumbrando sus sentidos, tocando su corazón y estimulando su mente (Widdis, 2001). Por lo tanto, promocionar la experiencia requiere una expresión más creativa por parte de las empresas. Las campañas de promoción tradicionales se centran más en las razones por la que la gente hace las cosas y menos en los factores no racionales que la gente tiene en cuenta a la hora de tomar sus decisiones.

Para los turistas, el principal requisito de un viaje agradable es que sea un viaje de experiencias (Botterill y Crompton, 1996). De hecho, hoy en día los turistas buscan experiencias únicas, innovadoras e imaginativas (Azevedo, 2009). En consecuencia, los destinos turísticos deben centrarse en los recuerdos agradables y asociaciones imaginadas por los turistas (Sternberg, 1997:951). Pine y Gilmore (1999) y Gilmore y Pine (2002) sugieren que promocionar experiencias requiere de una estrategia de marketing única que involucre a los clientes con una participación personal y de manera memorable.

A continuación se ejemplifica el desarrollo de marketing de experiencias a través de uno de sus instrumentos más paradigmáticos: los eventos. Los eventos integran al turista en su dinámica (involucración en su participación), y además, constituyen una herramienta de comunicación más allá de la experiencia del evento, incorporando la marca del destino y la experiencia global propuesta por dicha marca, generando una elevada memorabilidad. 
Desde los trabajos pioneros en la literatura turística sobre los eventos, comenzados en los años 80 por el profesor Getz, la creciente importancia de los eventos y festivales como productos turísticos no ha dejado de crecer. Tanto los eventos especiales como los «megaeventos» pueden aportar interesantes beneficios: elevar el número de turistas, aumentar la cobertura en los medios de comunicación y el prestigio, elevar el impacto económico para la comunidad de acogida o destino, mejorar la satisfacción del visitante, limitar el efecto de la estacionalidad de un destino turístico, aumentar los incentivos para atraer a nuevos mercados y atraer clientes que visitan por primera vez el destino, mejorar las tasas de ocupación en los hoteles, aumentar la estancia media, etc. (Getz, 1997).

Muchos autores coinciden a la hora de analizar el impacto económico derivado de los eventos (Perles, 2006; Seaman 2003; y Kasimati, 2003) destacando tres tipos de impactos o efectos que se pueden medir en función de los flujos de los agentes analizados: los efectos directos que se corresponden a los gastos realizados por la actividad o la institución organizadora en diferentes conceptos (salarios, compras, alquileres, ejecución de programas, etc.) en el área geográfica de referencia y en un período de tiempo determinado); los efectos indirectos, definidos como los gastos que realizan los visitantes o espectadores como consecuencia del evento (alojamiento, restauración, transporte, compras, entradas, etc.); y los efectos inducidos, entendiendo por los mismos todas aquellas repercusiones no contabilizadas en las categorías anteriores y que se difunden o amplían por el resto del sistema económico, dentro y fuera del espacio de referencia.

Los eventos ayudan a aumentar el atractivo de un destino, como una parte clave de la cartera de productos del mismo. Marchena y Repiso (1999) afirman que la tendencia al aumento de la demanda de los eventos, mega-eventos y otras actividades culturales, será evidente no solo en aquellos lugares que se han configurado tradicionalmente como un destino turístico, sino también en destinos emergentes. Los eventos han evolucionado a lo largo de las últimas décadas, pasando de ser meros productos diseñados para agregar a la cartera de atractivos que ofrece el destino, a convertirse en herramientas de marketing estratégico, cuyo propósito es no sólo atraer a los turistas, sino ayudar a desarrollar la notoriedad de los destinos (países, regiones o ciudades) que los acogen, así como el posicionamiento y la marca de los mismos. Los tipos de eventos pueden variar ampliamente, ya que incluyen festivales, ferias, eventos deportivos, eventos de arte, y también eventos de negocios, tales como exhibiciones, exposiciones, meetings, eventos educativos, carnavales y otros (Zerpa y Lunar, 2008; y García y Such, 2010). Debido a que son únicos, los festivales y los eventos, ya sean regionales, nacionales o internacionales, «son noticia», y por lo tanto atraerán el protagonismo mediático tanto en los medios tradicionales como en Internet.

Los destinos desean ser asociados con los eventos que poseen los atributos que el destino quiere adoptar (Moreno-Gil y Ritchie, 2009). Sin embargo, resulta complicado realizar la evaluación de los impactos económicos resultantes de los eventos, siendo más fácil para los destinos argumentar beneficios genéricos no cuantificados de imagen y marca. De hecho, el principal beneficio que los destinos pueden obtener de los eventos es la exposición de la marca y el desarrollo de la imagen (Dimanche, 2002). Un papel de los gerentes del destino es seleccionar, apoyar, u organizar eventos que contribuyan consistentemente a la comunicación de la imagen y atributos de la marca de los destinos. Los 
eventos son fuertes herramientas de comunicación y los responsables del destino deben asegurarse de que los eventos como experiencias y sus atributos estén bien alineados con la estrategia general de marketing del destino.

Para el visitante, un evento especial es una oportunidad de una experiencia fuera del rango normal de sus decisiones o más allá de la experiencia cotidiana. Los eventos turísticos tienen la particularidad de ofrecer a sus clientes un escenario en donde puede llegar a ser ellos mismos los actores, por lo tanto, creando experiencias valiosas y memorables. La poderosa combinación de eventos como constructores de la imagen para el destino, y de creadores de experiencias para entregar a los visitantes, contribuye a hacer de los eventos una de las estrategias clave para ayudar a los destinos a obtener una ventaja competitiva. Sin embargo, desarrollar una fuerte imagen de marca para un destino lleva años, y para que los eventos contribuyan de manera significativa a este esfuerzo, debe usarse un enfoque de desarrollo del evento coherente e integrado, en consonancia con los objetivos del destino y en relación a la experiencia a desarrollar y promocionar.

En este último epígrafe se ha enfatizado la importancia de desarrollar un marketing de experiencias, y prestar especial atención a los eventos como catalizadores experienciales, siendo éstos un elemento aglutinador y básico en la comunicación del destino, debiéndose entender cada fase del turista en el destino, y cada interacción, como un pequeño micro evento que necesita ser potenciado y sobre el que debe establecerse una mejora continua.

\section{CONCLUSIONES PARA EL DISEÑO DE EXPERIENCIAS TURÍSTICAS DE ÉXITO}

Este trabajo ha analizado el concepto y la naturaleza de la experiencia turística, habiendo identificado una serie de dimensiones que están presentes en las experiencias turísticas. También se han resaltado las diferentes metodologías existentes para su análisis e investigación, concluyéndose con la necesidad de trabajar con metodologías mixtas. Adicionalmente, se ha analizado la herramienta central de comunicación de un destino experiencial: la marca, identificándose las fases para su estructuración en un proceso dinámico donde la marca debe configurarse partiendo de la experiencia del turista, pero también aporta a la propia experiencia del mismo. Finalmente, se ha estudiado el desarrollo de marketing de experiencias para su adecuada comunicación, con un desarrollo específico del caso de los eventos turísticos, como paradigma de dicha promoción de experiencias.

A continuación, y fundamentándose en los análisis previos, se ha realizado un esfuerzo de estructurar de forma sintética los elementos clave para el diseño de una experiencia turística memorable y se expone un sencillo esquema metodológico de aplicación, integrando la perspectiva de gestión del destino y la del turista.

La filosofía de Pine y Gilmore de «el trabajo es teatro, y la actividad comercial un escenario» ofrece la base para el marco de gestión utilizado para implementar su «economía de la experiencia» donde los gerentes deben entender que convertir cada interacción en una experiencia es la premisa clave, conformando la suma de todas las interacciones una experiencia memorable para el turista. Así, Ford y Heaton (2000) desarrollan la gestión de la experiencia del cliente en hostelería, en la denominada «guestology», definida como la diferencia entre la calidad que el cliente espera y la calidad que el cliente obtiene después del consumo. 
Ya previamente, el concepto de semillas de la experiencia planteado por Csikszentmihalyi, se desarrolló con Berry (1981) y sus colaboradores (Zeithaml et al., 1985), con el éxito de su modelo SERVQUAL de calidad de la experiencia de servicio, como importante catalizador de una mayor atención hacia los aspectos intangibles de la experiencia en la base del pensamiento del marketing. Esta continua evolución en la naturaleza de la experiencia nos lleva a distinguir entre diferentes tipologías que, conceptualmente, parecen formar un camino evolutivo de la misma: la experiencia básica, la experiencia satisfactoria, la experiencia de calidad, la experiencia extraordinaria y la experiencia memorable. Diseñar una experiencia memorable es un aspecto clave, como se argumenta ampliamente en este trabajo y exige una doble aproximación: desde la perspectiva de la gestión del destino, y desde la del turista.

\subsection{La perspectiva de la gestión del destino}

Como se expuso previamente, la imagen turística de los destinos se construye en base a un referente real conformado por el espacio físico que ocupa el destino y las características ambientales, económicas y socioculturales que se establecen. Sobre esa base real se articula toda una imaginería turística, con el fin de enmascarar lo más desagradable, al mismo tiempo que recrea, e idealiza los aspectos más interesantes del destino. En el proceso de creación de la imagen turística de los destinos se distorsionan las características reales de éstos. Es el proceso de «hiperrealidad» teorizado por Eco (1986) en Travels in Hyperreality, o por Baudrillard (1993) en La Precession des simulacres. Por consiguiente, cada fase del proceso vacacional del turista debe ser considerada por los gerentes del destino, ya que puede ser alterada, integrando los elementos intangibles y de imagen que convierten dicha interacción en algo memorable.

Disneylandia es, sin duda, el ejemplo por excelencia en la que una invención acaba superando la realidad (Martín, 2003). Este concepto está muy desarrollado por empresas como Disney y Starbucks, conscientes de que una ventaja competitiva sostenible sólo se puede alcanzar dándole al cliente una experiencia única y memorable. Como ejemplo de la adopción comercial del concepto «Gestión de la Experiencia del Cliente» (CEM), se ha desarrollado esta metodología para controlar todos los contactos que un cliente tiene con la empresa o destino. De esta manera, la cadena de gestión está convergiendo con la cadena de consumo. Se analiza punto por punto cada fase del proceso y cada interacción, tratando de identificar elementos de mejora.

\subsection{La perspectiva del turista}

Por el lado del turista, ha surgido un enfoque sobre la experiencia en respuesta a las limitaciones de ver el comportamiento del consumidor sólo en términos de procesamiento de la información cognitiva y como un proceso lógico y secuencial. Como dicen Holbrook y Hirschman (1982), las experiencias son subjetivas, estados emocionales cargados de significado simbólico, y el consumo es hedonista no utilitario, especialmente en situaciones de ocio.

Las dos líneas de investigación en la literatura sobre las experiencias turísticas: la perspectiva gerencial y la del consumidor, suelen estar de acuerdo en considerar al turista como 
un objeto final, pero también como el co-creador del producto (Prahalad y Ramaswamy, 2004). El objetivo de las experiencias extraordinarias es el crecimiento y la realización personal (Arnould y Price, 1993) que conduce a la transformación del turista. Es mediante el diseño de un escenario o del espacio para que esto suceda, que una empresa o destino puede atraer y retener a los turistas.

El diseño y desarrollo de una experiencia turística debe incorporar este enfoque de análisis de procesos gerencial, pero parece insuficiente para abordar toda la complejidad de la misma. Así, también debe integrar la perspectiva del turista. Como las experiencias son muy subjetivas, una experiencia significativa nunca puede ser garantizada. Sin embargo, se pueden crear las condiciones ideales para recrear experiencias memorables. Considerando que los estudios teóricos no suelen proveer de un instrumento operacional para aplicar el diseño de experiencias (Mehmetoglu y Engen, 2011), a continuación se muestra un sencillo instrumento práctico para analizar, comprender y diseñar los elementos que componen una experiencia memorable, integrando la perspectiva de gestión del destino y la del turista.

La figura 1 (matriz de diseño de la experiencia) integra ambas perspectivas. En el eje vertical se consideran las fases desde la perspectiva de gestión del destino, con las distintas etapas en las que se divide el proceso del disfrute vacacional del turista en el destino. Tomando el proceso de consumo tradicional) de un turista en un destino de sol y playa (Moreno et al., 2002, se han identificado las siguientes fases genéricas: información y reserva (en origen), llegada, transporte, check-in en el hotel, alojamiento en la habitación, consumo de ocio en el destino, transporte en el destino, compras, alimentación, salida, y retorno a casa. Estas fases pueden desarrollarse más en detalle, e incluso desglosarse los diferentes elementos que las componen, llegándose a un nivel de análisis sumamente minucioso. Así por ejemplo, en la figura 1 se muestra con cierto nivel de desglose, y a modo de ejemplo, la fase de check-in en el hotel: bienvenida, solicitud de documentación, entrega de llaves e información general, indicaciones para acceder a la habitación. Esta descomposición debe hacerse para cada fase si se desea un análisis completo de la experiencia.

En el eje horizontal se integra la perspectiva del turista, enumerando los aspectos identificados en la literatura como necesarios para recrear una experiencia memorable: involucración del turista, shock sensorial, autenticidad, diversión, sociabilidad, personalización, y autodescubrimiento y trasformación.

A continuación, la metodología propuesta plantea cruzar ambos ejes y someter cada fase, y cada subfase del destino (eje vertical), a un análisis participativo y reflexivo de posibles mejoras a incorporar en la experiencia, tratándose uno por uno las posibilidades de incorporar los elementos experienciales del individuo (eje horizontal).

Ilustrando de forma breve nuevamente el caso de la fase de check-in, y en concreto la subfase de entrega de laves al cliente e información general, debemos plantearnos posibles mejoras en relación a cada uno de los aspectos que incorporan memorabilidad a la experiencia y que aparecen en el eje horizontal. Así, se recogen mejoras de las personas del equipo que participen en la dinámica sobre cómo mejorar la involucración del turista en la entrega de la información del resort y el destino, incorporando elementos de la naturaleza del destino y vinculando esa información con los aspectos internos y propios 
Figura 1

MATRIZ DE DISEÑO DE LA EXPERIENCIA

\begin{tabular}{|c|c|c|c|c|c|c|c|c|c|c|}
\hline & & Destino: & & & & Seg & gme & nto & & \\
\hline FASES DEL VIAJE & & & & & & & & & & \\
\hline FASES & Código & SUBFASES & 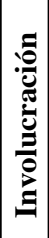 & 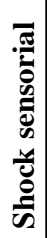 & 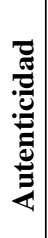 & 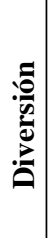 & 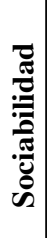 & 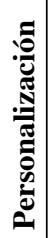 & 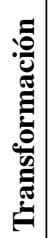 & $\stackrel{\mathscr{E}}{0}$ \\
\hline Información y Reserva & & & & & & & & & & \\
\hline Llegada & & & & & & & & & & \\
\hline Transporte & & & & & & & & & & \\
\hline & & Bienvenida & & & & & & & & \\
\hline & & Solicitud documentación & & & & & & & & \\
\hline 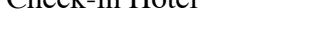 & & Entrega de llaves e información & & & & & & & & \\
\hline & & Indicaciones acceso habitación & & & & & & & & \\
\hline Alojamiento & & & & & & & & & & \\
\hline Consumo de Ocio & & & & & & & & & & \\
\hline Transporte en Destino & & & & & & & & & & \\
\hline Compras & & & & & & & & & & \\
\hline Alimentación & & & & & & & & & & \\
\hline Salida y Retorno a Casa & & & & & & & & & & \\
\hline OBSERVACIONES: & & & & & & & & & & \\
\hline
\end{tabular}

Fuente: Elaboración propia.

del individuo. Una vez anotadas todas las posibles mejoras propuestas, se continúa con el segundo aspecto del eje horizontal aplicado a la misma fase, esto es, cómo mejorar el shock sensorial en la entrega de la información y llaves, incorporando los cinco sentidos en dicho proceso y generando sorpresa sensitiva y un estímulo al cliente. Se continúa con el siguiente aspecto, y cómo incorporar autenticidad en dicha subfase, y que los elementos propios e identitarios del destino entren en juego, ya sea con el material empleado para dar la información, su diseño, las palabras usadas, etc. El siguiente aspecto es el de diversión, tratando de incorporar, por ejemplo, elementos de gamificación en dicha subfase. A continuación se sugieren mejoras que ayuden a añadir mayor sociabilidad en la entrega de información y llaves, dando lugar a posibilidades de creación de comunidad y 
de compartición, por ejemplo, a través de las nuevas tecnologías, propiciando interacción entre el producto y el cliente, con el personal de servicio, los otros clientes, el entorno, sus amigos, etc. Le sigue la personalización, que más allá de considerar el nombre del turista, pasa, por ejemplo, por añadir sus preferencias en la información facilitada y darle cierta posibilidad de elección al turista. Finalmente, en el autodescubrimiento y transformación, se trata de cómo incorporar aprendizaje en dicha subfase.

A modo de check-list, debemos ir analizando cada fase y subfase, y las posibilidades de mejora para cada criterio de servicio que aporta memorabilidad a la experiencia, de modo que la suma de sugerencias de mejora conforman una matriz de diseño de experiencia, que transforma un servicio rutinario en el destino en una experiencia memorable para el turista. Este esquema de trabajo, acompañado de las técnicas adecuadas, permite generar una gran cantidad de oportunidades de mejora para el diseño de una experiencia memorable, que a través de un marketing de experiencias se traduce en un destino de experiencias y en la comunicación de su marca. Obviamente, la combinación de elementos de mejora variará en función del contexto y contenido de la experiencia, y el segmento o persona a la que se dirija, pero la matriz supone un sencillo instrumento de aplicación para buscar las propuestas adecuadas a cada caso.

Finalmente, la aplicación de esta metodología de matriz de diseño de la experiencia turística, vinculada a la marca destino y el marketing de experiencias, significa no sólo considerar la investigación como un instrumento de análisis y mejora, sino como parte integrante del propio proceso de promoción de la empresa o destino. Se trata de un verdadero marketing de experiencias integrando la sociedad del conocimiento y la investigación, donde los turistas co-analizan la experiencia, la co-crean y la comparten con otros turistas potenciales.

\section{BIBLIOGRAFÍA}

AAKER, D.A. (1991): Managing brand equity: capitalizing on the value of a brand name. New York: The Free Press.

AAKER, J.L. (1997): «Dimension of Brand Personality», Journal of Marketing Research, $\mathrm{n}^{\mathrm{o}} 34$ (August), pp. 347-56.

ABRAHAMS R. (1986): «Ordinary and extraordinary experience» en The Anthropology of Experience. TURNER y BRUNER (eds.). Illinois, University of Illinois, Press: Urbana and Chicago, pp. 45-72.

AHO, S.K. (2001): «Towards a general theory of touristic experiences: Modeling experience process in tourism», Tourism Review, vol. 56, n 3/4, pp. 33-37.

ALLOZA, A. (2008): «Brand Engagement and Brand Experience at BBVA, The Transformation of a 150 Years Old Company», Corporate Reputation Review, vol. 11, $\mathrm{n}^{\circ}$ 4, pp. 371-381.

AMBLER, T.; BHATTACHARYA, C.B.; EDELL, J.; KELLER, K.L.; LEMO, K.N. y MITTAL, V. (2002): «Relating brand and customer perspectives on marketing management», Journal of Service Research, vol. 5, n ${ }^{\circ}$ 1, pp. 13-25.

ARNOULD, E.J.; PRICE, L.L. (1993): «River magic: Extraordinary experience and the extended services encounter», Journal of Consumer Research, vol. 20, n 1, pp. 24-45. 
AZEVEDO, A. (2009): «Designing unique and memorable experiences: Co-creation and the «surprise» factor», en Proceedings of X Congresso Internacional de Turismo de Leiria e Oeste, Leiria, Portugal.

BAUDRILLARD, J. (1993): «The precession of simulacra» (P. FOSS, P. PATTON \& P. BEITCHMAN, Trans.), en J. NATOLI \& L. HUTCHEON (Eds.), A postmodern reader (pp. 342-375). Albany: State University of New York Press. (Original work published 1983).

BERRY, LL. (1981): «The Employee as Customer», Journal of Retail Banking, $\mathrm{n}^{\circ}$ 3(March), pp. 33-40.

BERRY, L.L.; CARBONE, L.P. y HAECKEL, S.H. (2002): «Managing the Total Customer Experience», MIT Sloan Management Review, vol. 43, n 3, pp. 85-89.

BIGNÉ, E.; FONT, X. y ANDREU, L. (2000): Marketing de destinos turísticos análisis y estrategias de desarrollo. Madrid: Esic.

BIGNÉ, J.E.B., SÁNCHEZ, M.I. y SÁNCHEZ, J. (2001): «Tourism image, evaluation variables and after purchase behavior: inter-relationship», Tourism Management, vol. 22, pp. 607-16.

BLAIN, C.; LEVY, S.E. y RITCHIE, B. (2005): «Destination branding: insights and practices from destination management organizations», Journal of Travel Research, vol. 43, no 4, pp. 328-338.

BOORSTIN (1964): The image: A guide to pseudo-events in America, New York: Harper \& Row.

BOTTERILL, T.D. y CROMPTON, J.L. (1996): «Two case studies exploring the nature of the tourist's experience», Journal of Leisure Research, vol. 28, $\mathrm{n}^{\circ}$ 1, pp. 57-82.

BRAKUS, J.J., SCHMITT, B.H. y ZARANTONELLO L. (2009): «Brand experience; What is it? How is it measured? Does it affect loyalty?», Journal of Marketing, May 2009, pp. 52-68.

BRAKUS, J.J.; SCHMITT, B.H. y ZHANG, S. (2008): «Experiential attributes and consumer judgments», en Handbook on Brand and Experience Management, BERND H. SCHMITT and DAVID ROGERS, eds. Northampton, MA: Edward Elgar.

CAI, L.A. (2002): «Cooperative branding for rural destinations», Annals of Tourism Research, vol. 29, n 3, pp. 720-742.

CAMISÓN, C. y MONFORT, V.M. (1998): «Estrategias de reposicionamiento para destinos turísticos maduros el caso de la Costa Blanca», Estudios Turísticos, n ${ }^{\circ} 135$, pp. 5-28.

CARBAllo, M.; ARAÑA, J.; LEÓN, C.; GONZÁlEZ, M. y MORENO, S. (2011): «Valoración Económica de la Imagen de un Destino», Pasos. Revista de Turismo y Patrimonio Cultural, vol. 9, nº 1, pp. 1-14.

CARLZON, J. (1987): Moments of Truth. Harper \& Row, New York.

CARROLL, B. y AHUVIA, A. (2006): «Some Antecedents and Outcomes of Brand Love», Marketing Letters, vol. 17, $\mathrm{n}^{\circ}$ 2, pp. 79-89.

COHEN, E. (1979): «A phenomenology of tourist experiences», Sociology, $\mathrm{n}^{\circ} 13$, pp. 179-201.

COOPER, C. y JACKSON, S. (1989): «Destination life cycle. The Isle of Man case study», Annals of Tourism Research, vol. 16, n 3, pp. 377-398. 
CROMPTON, J.L. (1979): «An assessment of the image of Mexico as a vacation destination and the influence of geographical location upon that image», Journal of Travel Research, vol. 17, $\mathrm{n}^{\mathrm{O}}$ 4, pp. 18-23.

CSIKSZENTMIHALYI, M. (1975): «Beyond boredom and anxiety: The experience of play in work and games», en Jossey-Bass Publishers: San Francisco.

CSIKSZENTMIHALYI, M. (2000): «The Costs and Benefits of Consuming», Journal of Consumer Research, vol. 27, n 2, pp. 267-272.

CUTLER, S.Q. y CARMICHAEL, B.A. (2010): «The dimensions of the tourist experience», en M. MORGAN, P. LUGOSI, and J. R. B. RITCHIE (Eds.), The tourism and leisure experience. Consumer and managerial perspectives (pp. 3-26). Bristol, UK: Channel View Publications.

DELGADO BALLESTER, E.; MUNUERA ALEMÁN, J.L. y YAGÜE GUILLÉN, M.J. (2003): «Development and validation of a brand trust scale», International Journal of Market Research, vol. 45, n 1, pp. 35-53.

DIMANCHE, F. (2002): «The contribution of special events to destination brand equity», en K. W. WÖBER (Ed.) City Tourism 2002: Proceedings of European Cities Tourism's International Conference, pp. 73-80. Vienna: Springer.

ECO, U. (1986): Travels in hyperrality. London. Ed. Picador.

EKINCI Y, HOSANY S. (2006): «Destination personality: an application of brand personality to tourism destinations», Journal of Travel Research, vol. 45, n 2, pp. 127-139.

FORD, R.C. y HEATON, C.P. (2000): Managing the guest experience in hospitality. Delmar Learning: Clifton Park, NY.

FRIDGEN, J. D. (1984): «Environmental psychology and tourism», Annals of Tourism Research, vol. 11, n ${ }^{\circ}$, pp. 19-39.

GARCÍA, J. M. y SUCH, M.J. (2010): «Influencia de los mega-eventos en la oferta alojativa de un destino: Los juegos olímpicos», Revista de Análisis Turístico. AECIT. $\mathrm{n}^{\circ}$ 10 , pp 45-55.

GETZ, D. (1997): Event Management and Event Tourism. New York: Cognizant Communication.

GILMORE, J. y PINE, B. (2002): The experience is the marketing. Aurora, OH: Strategic Horizons LLP.

GOODALL, B. y ASWORTH, G. (1988): Marketing in the tourism industry: the promotion of destination regions. London: Groom Helms.

HANKINSON, G. (2004): «The brand images of tourism destinations: a study of the saliency of organic images», Journal of Product and Brand Management, vol. 13, $\mathrm{n}^{\mathrm{o}}$ 1, pp. 6-14.

HARRIS, C. y ATELJEVIC, I. (2003): «Perpetuating the male gaze as the norm: Challenges for "her" participation in business travel», Tourism Recreation Research, vol. $28, \mathrm{n}^{\mathrm{o}} 2$, pp. 21-30.

HARRISON, J. (2001): «Thinking about tourists», International Sociology, vol. 16, pp. 159-172.

HAVLENA, J. y HOLBROOK, B. (1986): «The varieties of consumption experience: comparing two typologies of emotion in consumer behavior», Journal of Consumer Research, $\mathrm{n}^{\circ} 13$, pp. 394-404. 
HIRSCHMAN, E.C. (1984): «Experience seeking: A subjectivist perspective of consumption», Journal of Business Research, vol. 12, n 1, pp. 115-136.

HOLBROOK, M.B. y HIRSCHMAN, E.C. (1982): «The experiential aspects of consumption: Consumer fantasies, feelings, and fun», Journal of Consumer Research, vol. 9, $\mathrm{n}^{\mathrm{o}} 2$, pp. 132-140.

HOLBROOK, M. B. (2000): «The Millennial Consumer in the Texts of Our Times: Experience and Entertainment», Journal of Macromarketing, vol. 20, $\mathrm{n}^{\circ} 2$, pp. 178-92.

HUDSON, S.; WANG, Y. y MORENO-GIL, S. (2011): «The influence of a Film on Destination Image and the Desire to Travel: a Cross-Cultural Comparison», International Journal of Tourism Research, vol. 13, pp. 177-190.

IACVB, Kovaleski, D., and G. Sherwin. (2005): Destination Brand Science. Washington, DC: Destination Marketing Association International (formerly known as International Association of Convention \& Visitors Bureau).

JACKSON, M.S.; WHITE, G.N. y SCHMIERER, C.L. (1996): «Tourism experiences within an attributional framework», Annals of Tourism Research, vol. 23, n 4, pp. 798-810.

JENNINGS, G. y NICKERSON, N.P. (2006): Quality Tourism Experiences. Burlington, MA: Elsevier Butterworth-Heinemann.

KASIMATI, E. (2003): «Economic aspects and the Summer Olympics: a review of related research», International Journal of Tourism Research, vol. 5, n ${ }^{\circ}$ 6, pp. 433-444.

KEISER, J.D. (1998): «Hospitality and tourism: a rhetorical analysis and conceptual framework for identifying industry meanings», Journal of Hospitality and Tourism Research, vol. 22, $\mathrm{n}^{\mathrm{O}} 2$, pp.15-128.

KELLER, K.L. (1993): «Conceptualizing, measuring and managing customer-based brand equity», Journal of Marketing, vol. 57, pp. 1-22.

KNAPP, D. y SHERWIN, G. (2005): Destination Brand Science. International Association of Convention and Visitor Bureaus: Washington, DC.

KOTLER, P. (1997): Marketing management: analysis, planning, implementation and control. 9th edition. Prentice Hall, Upper Saddle River, NJ.

LARSON, R. y CSIKSZENTMIHALYI, M. (1983): «The experience sampling method», en New Directions for Naturalistic Methods in the Behavioral Sciences, REIS, H. (ed.). Jossey-Bass: San Francisco; 41-56.

LEE, Y.; DATTILO, J. y HOWARD, D. (1994): «The complex and dynamic nature of leisure experience», Journal of Leisure Research, vol. 26, n 3, pp. 195-211.

LEIGHTON, D. (2007): «Step back in time and live the legend: experiential marketing and the heritage sector», International Journal of Nonprofit and Voluntary Sector Marketing, vol. 12, n ${ }^{\circ}$ 2, pp. 117-125.

LOW, G.S. y LAMB, C.W. (2000): «The measurement and dimensionality of brand associations», Journal of Product and Brand Management, vol. $9 \mathrm{n}^{\circ}$ 6, pp. 350-68.

MAcCANNELL, D. (1973): «Staged authenticity: Arrangements of social space in tourist settings», The American Journal of Sociology, vol. 79, n 3, pp. 589-603.

MARCHENA, M. y REPISO, F. (1999): «Turismo cultural: El caso de Sevilla», Cuadernos de Turismo, $\mathrm{n}^{\mathrm{o}} 4$, pp. 33-50. 
MARTÍN, B. (2003): «La imagen turística de las regiones insulares las islas como paraísos», Cuadernos de Turismo, $\mathrm{n}^{\circ}$ 11, pp. 127-137.

MARTÍNEZ, E. y CHERNATONY, L. (2004): «The effect of brand extension strategies upon brand image», Journal of Consumer Marketing, vol. 21, n 1, pp. 39-50.

McALEXANDER, J.H.; SCHOUTEN, J.W. y KOENIG, H.F. (2002): «Building Brand Community», Journal of Marketing, 66 (January), pp. 38-54.

MEHMETOGLU, M. y ENGEN, M. (2011): «Pine and Gilmore's concept of experience economy and its dimensions: An empirical examination in tourism», Journal of Quality Assurance in Hospitality and Tourism, 12, $\mathrm{n}^{\circ}$ 4, pp. 237-255.

MEHRABIAN, A. y RUSSELL, J. (1974): An approach to environmental psychology, Cambridge, Massachusetts, MA: MIT Press.

MORENO, G.S.; CELIS, S.D. y AGUIAR, Q.T. (2002): «Análisis de la satisfacción del turista de paquetes turísticos respecto a las actividades de ocio en el destino: El caso de Republica Dominicana», Cuadernos de Turismo, no 9, pp. 67-84.

MORENO-GIL, S. y RITCHIE, B.J.R. (2009): «Understanding the Museum Image Formation Process: A Comparison of Residents and Tourists», Journal of Travel Research, vol. 47, pp. 480-493.

MORGAN, N; PRITCHARD, A. y PIGGOTT, R. (2003): «Destination branding and the role of the stakeholders: the case of New Zealand», Journal of Vacation Marketing, vol. $9, n^{\circ} 3$, pp. 285-299.

MORGAN, N.; PRITCHARD A. y PIGGOTT, R. (2002): «New Zealand, 100\% pure: the creation of a powerful niche destination brand», Journal of Brand Management, vol. 9, $\mathrm{n}^{\mathrm{o}}$ 4/5, pp. 335-354.

NICKERSON, N.P.; KERSTETTER, D.; BRICKER, K. y ANDERECK, K. (2004): «Understanding visitors' experiences: methodological comparisons», en Measuring the Tourism Experience: When experience rules, what is the metric of success? Conference Proceedings of the Travel and Tourism Research Association, Montreal, Quebec, June 20-23.

NOY, C. (2004): «This trip really changed me: Backpackers' narratives of self-change», Annals of Tourism Research, vol. 31, n 1, pp. 78-102.

O’DELL, T. y BILLING, P. (2005): Experiencescapes: tourism, culture, and economy, Copenhagen Business School Press: Copenhagen, Denmark.

OBIOL MENERO, E.M. (2002): «Marcas turísticas y territorio. Un análisis geográfico del turismo valenciano», Cuadernos de Turismo, n 9, pp. 85-101.

OTTO, J.E. y RITCHIE, J.R.B. (1996): «The service experience in tourism», Tourism Management, vol. 17, $\mathrm{n}^{\circ}$ 3, pp. 165-174.

PEARCE, P. L. y FOSTER, F. (2007): «A “university of travel”: Backpacker learning», Tourism Management, $\mathrm{n}^{\circ} .28$, pp. 1285-1298.

PERLES, J. F. (2006): «Análisis del impacto económico de eventos: una aplicación a fiestas populares de proyección turística», Cuadernos de Turismo, n 17, pp. 147-166.

PETKUS, E. JR. (2004): «Enhancing the application of experiential marketing in the arts», International Journal of Nonprofit and Voluntary Sector Marketing, vol. $9, \mathrm{n}^{\circ}$ 1, pp. 49-56.

PINE, B.J. y GILMORE, J.H. (1999): The experience economy: Work is theatre and every business a stage, Harvard Business School Press: Boston. 
PINE, B.J. y GILMORE, J.H. (1998): Welcome to the experience economy, Harvard Business Review, pp. 97-107.

PRAHALAD, C.K. y RAMASWAMY, V. (2004): «Co-creating unique value with customers», Strategy and Leadership, vol. 32, n 3, pp. 4-9.

PRESENZA, A.; MORENO, G.S. y LOBO, L.A. (2007): «El funcionamiento de las organizaciones de marketing de destinos regionales. El caso de la organización regional de turismo «Abruzzo Promozione Turismo» (Italia)», Cuadernos de turismo, nº19, pp.133-146.

RICHARDS, G. y WILSON, J. (2006): «Developing creativity in tourist experiences: A solution to the serial reproduction of culture?», Tourism Management, vol. 27, $\mathrm{n}^{\circ} 6$, pp. 1209-1223.

RITCHIE, J.R.B. y RITCHIE, R.J.B. (1998): «The branding of tourism destinations: past achievements and future challenges» en Annual Congress of the International Association of Scientific Experts in Tourism (AIEST), September 1998, Marrakesh, Morocco: 89-116.

RITCHIE, B. J. R. y HUDSON, S. (2009): «Understanding and Meeting the Challenges of Consumer/Tourist Experience Research», International Journal of Tourism Research, vol. 11, no 2, pp. 111-126.

ROBERTSON, H. (2007): «At last a definition of experiential marketing», Marketing Week, January 18: 21.

RYAN, C. (1997): The Tourist Experience: A New Introduction. Cassell: London.

RYAN, C. (1995): Researching Tourist Satisfaction: Issues, Concepts, Problems, London: Routledge.

SCHMITT, B. (2003): Customer Experience Management, New York: The Free Press.

SCHMITT, B. (1999): «Experiential marketing», Journal of Marketing, $\mathrm{n}^{\circ}$ 15, pp. 53-67.

SEAMAN, B. (2003): «Economic impact of the arts» en TOWSE R. (2003) A Handbook of Cultural Economics, 27, pp. 224-231.

SEDDIGHI, H. R. y A. THEOCHAROUS, L. (2002): «A model of tourism destination choice: A theoretical and empirical analysis», Tourism Management, vol. 23, $\mathrm{n}^{\circ}$ 5, pp. 475-87.

SERAROLS, C. y ROYO, M. (2005): «El turismo rural-cultural: Un modelo de gestión del marketing turístico a nivel local basado en la medida de la imagen del destino», Cuadernos de Turismo, no ${ }^{\circ}$ 16, pp. 197-222.

STERNBERG, E. (1997): «The iconography of the tourism experience», Annals of Tourism Research, vol. 24, $\mathrm{n}^{\circ}$ 4, pp. 951-969.

TASCI, A.D.A. y KOZAK, M. (2006): «Destination brands vs. destination images: Do we know what we mean?», Journal of Vacation Marketing, vol. 12, nº 4, pp. 299-317.

THOMSON, M.; MAcINNIS, D. J. y PARK, C. W. (2005): «The ties that bind: Measuring the strength of consumers' emotional attachments to brands», Journal of Consumer Psychology, vol. 15, no 1, pp. 77-91.

TSAUR, S.H; CHIU, Y.T. y WANG, C.H. (2006): «The visitors behavioural consequences of experiential marketing: an empirical study on Taipei Zoo», Journal of Travel and Tourism Marketing, vol. 21, $\mathrm{n}^{\mathrm{o}}$ 1, pp. 47-64.

VOSS, C. (2004): Trends in the Experience and Service Economy, the Experience Profit Cycle, London School of Business, pp 1-39. 
WELLS, W.; BURNETT, B. y MORIARTY, S. (2006): Advertising principles and practice, Prentice Hall: Englewood Cliffs, NJ.

WIDDIS, P. (2001): «Bringing brands to life: experiential marketing works by touching consumers' hearts», Marketing Magazine, vol. 106, $\mathrm{n}^{\circ}$ 2, pp. 18.

WILLIAMS, A. (2006): «Tourism and hospitality marketing: fantasy, feeling and fun», International Journal of Contemporary Hospitality Management, vol. 18, $\mathrm{n}^{\circ} 6$, pp. 482-495.

ZEITHAML, V.A.; PARASURAMAN, A. y BERRY, LL. (1985): «Problems and strategies in services marketing», Journal of Marketing, 49 (Spring), pp. 33-46.

ZERPA, J.C. y LUNAR, R.A. (2008): «Diagnóstico de los bienes del patrimonio históricocultural del municipio Zamora, como atractivos turísticos del Estado Aragua», Pasos, Revista de Turismo y Patrimonio Cultural, vol. 6 n 3, pp. 523-540. 